\title{
TITLE:
}

\section{Phase and group velocity matching for cumulative harmonic generation in Lamb waves}

AUTHOR(S):

Matsuda, Naoki; Biwa, Shiro

CITATION:

Matsuda, Naoki ... [et al]. Phase and group velocity matching for cumulative harmonic generation in Lamb waves. Journal of Applied Physics 2011, 109(9): 094903.

\section{ISSUE DATE:}

2011

URL:

http://hdl.handle.net/2433/143568

RIGHT:

(C) 2011 American Institute of Physics 


\title{
Phase and group velocity matching for cumulative harmonic generation in Lamb waves
}

\author{
Naoki Matsuda and Shiro Biwa ${ }^{a)}$ \\ Department of Micro Engineering, Graduate School of Engineering, Kyoto University, Kyoto 606-8501, Japan
}

(Received 19 November 2010; accepted 25 February 2011; published online 5 May 2011)

\begin{abstract}
Owing to the enhanced sensitivity of nonlinear acoustic methods to material damage, the nonlinear Lamb wave propagation is pertinent to the nondestructive evaluation of platelike structures, and it is typically manifested as generation of higher harmonics. For dispersive waves such as Lamb waves, however, the cumulative growth of harmonics requires that the primary mode and the generated higher harmonic modes possess identical phase and group velocities. In this paper, this issue of the phase and group velocity matching in Lamb waves is explored based on a systematic analysis of the Rayleigh-Lamb frequency equations. The analysis shows that for certain values of the phase velocity, the Rayleigh-Lamb frequency equations are satisfied at equi-spaced frequencies which are multiples of the smallest. Such frequencies, together with the corresponding phase velocities and the Lamb modes, are determined analytically. Four such types of Lamb modes are identified: (i) Lamé modes, (ii) symmetric modes with dominant longitudinal displacements, (iii) intersections of symmetric and antisymmetric modes and (iv) extra Rayleigh modes. For the first three types, it is also established that the primary and the harmonic modes have the same group velocity, and that the surface motion of the plate is featured with vanishing vertical or horizontal displacements. In contrast to these three types, the fourth type only exists for a special range of the transverse to longitudinal wave speeds of the solid. This type is not featured with a common group velocity, and neither of the vertical or horizontal displacement vanishes on the plate surfaces. The obtained results are summarized as tables, and demonstrated graphically on the dispersion curves for aluminum as well as iron plates. (c) 2011 American Institute of Physics. [doi:10.1063/1.3569864]
\end{abstract}

\section{INTRODUCTION}

Nonlinear ultrasonic methods offer a promising means for nondestructive evaluation of structural integrity owing to their enhanced sensitivity to material degradation as compared to conventional techniques. In particular, the generation of higher harmonics by sinusoidal or narrow-band incident waves has been extensively studied in the light of characterization of plastic strains, fatigue damage, microcracking and other types of material damage. ${ }^{1-6}$ While most of foregoing studies on the acoustic harmonic generation dealt with nondispersive modes such as bulk and Rayleigh waves, the corresponding phenomenon in dispersive guided waves, such as Lamb waves in a plate, is currently receiving increasing attention for materials evaluation. ${ }^{7-12}$

Theoretically, the harmonic generation in Lamb wave propagation has been investigated based on the perturbation approach and the modal analysis technique. ${ }^{7-9}$ These studies have revealed that, in general, the propagation of an incident (primary) Lamb wave with a certain frequency does not always generate its higher harmonics in a cumulative fashion, i.e., the amplitudes of the harmonic Lamb modes do not grow proportionally with the propagation distance. This is due to the dispersive nature of Lamb waves which implies that the primary Lamb wave and its harmonics generally pos-

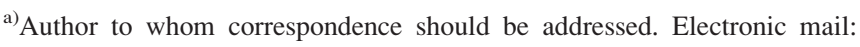
biwa@kuaero.kyoto-u.ac.jp.
}

sess different propagation velocities. The cumulative generation of higher harmonics is practically an important problem in order to measure the nonlinear effect with sufficient signal-to-noise ratio.

In order to achieve cumulative harmonic generation, Deng ${ }^{7,8}$ claimed that the primary and its harmonic Lamb modes should have an identical phase velocity (phase matching). The analysis was elaborated by de Lima and Hamilton, ${ }^{9}$ who pointed out the necessity of the so-called nonvanishing power transfer from the primary to the harmonic mode. Srivastava and Lanza di Scalea ${ }^{13}$ provided further analysis on this issue and discussed the existence of symmetric or antisymmetric mode in each order of the harmonics. Furthermore, some authors ${ }^{12,14,15}$ have argued that the group velocities of the primary and the harmonic modes should also be equal. In order to fulfill these requirements, the mode and the frequency of the primary Lamb wave to enable cumulative harmonic generation cannot be arbitrary. It is then of significant importance to understand what mode and frequency of Lamb waves can bring about the cumulative harmonic generation. In the above-mentioned studies, however, only some specific modes and frequencies have been demonstrated which exhibit this phenomenon. A comprehensive analysis of the Lamb modes and frequencies for cumulative harmonic generation deserves special attention from fundamental as well as practical points of view.

Recently, Müller et al. ${ }^{16}$ presented an analysis of the phase and group velocity matching as well as the nonzero 
power transfer for the cumulative growth of the second harmonics in Lamb waves. They identified five types of the primary Lamb modes that satisfy these conditions, two of them being of an exact nature and three being asymptotic. In this paper, the issue of phase and group velocity matching in Lamb waves is examined in a different perspective. Namely, a theoretical analysis is presented to derive the modes and frequencies of Lamb waves whose higher harmonics have the identical phase as well as group velocities. The present analysis shows that for certain values of phase velocity, a series of equi-spaced angular frequencies exist, satisfying the dispersion relations simultaneously. Therefore, the analysis is pertinent to the cumulative generation of not only second harmonics, but also higher harmonics of arbitrary orders.

The analysis in this paper is carried out in the spirit of the perturbation analysis employed in the foregoing studies, ${ }^{7-9}$ which enables us to discuss this issue based on the Rayleigh-Lamb frequency equations in the linear theory of Lamb wave propagation as outlined in Sec. II. Some properties of these equations are exploited in a systematic manner in Sec. III to derive the phase velocities for which the phase matching is possible. For the corresponding sets of equispaced frequencies, the matching of the group velocity is also examined. In Sec. IV, it is shown that the derived conditions for the phase velocity are indeed necessary for the phase matching. The derived results are summarized in Sec. V as four characteristic mode types. While two of these mode types correspond to those of exact matching shown by Müller et al. ${ }^{16}$ for the second-harmonic generation, the present analysis includes two other types which were not mentioned by them. Some results of other foregoing studies are shown to be included as special cases of the mode types characterized here. Discussions are also given on the characteristics of the surface motions of the plate in the Lamb modes exhibiting the phase matching. In Sec. VI, the phase and group velocity matching are demonstrated graphically on the dispersion curves for specific materials.

\section{RAYLEIGH-LAMB FREQUENCY EQUATIONS}

According to the perturbation approach to the wave propagation in an isotropic elastic plate assuming weak stress-strain nonlinearity, the analysis for the harmonic generation when a Lamb mode with a certain frequency propagates can be decomposed into two linearized problems corresponding to the unperturbed primary mode, and the harmonic mode subject to the excitation source due to the nonlinear contribution of the primary mode. Readers are referred to the foregoing papers ${ }^{7-9,13,16}$ for the explicit solution of these problems. In this situation, the phase matching can be discussed based on the Rayleigh-Lamb frequency equations for a linear and isotropic elastic plate (thickness $d=2 h$ ). For the present discussion, these equations are expressed in the following forms, namely,

$$
\begin{aligned}
\Omega_{\mathrm{S}}(\omega, k) \equiv & \left\{q^{2}-k^{2}\right\}^{2}\left(\frac{\sin q h}{q}\right) \cos p h \\
& +4 k^{2} p \sin p h \cos q h=0,
\end{aligned}
$$

for the symmetric (S) modes, and

$$
\begin{aligned}
\Omega_{\mathrm{A}}(\omega, k) \equiv & \left\{q^{2}-k^{2}\right\}^{2}\left(\frac{\sin p h}{p}\right) \cos q h \\
& +4 k^{2} q \sin q h \cos p h=0,
\end{aligned}
$$

for the antisymmetric (A) modes, where

$$
p=\sqrt{\left(\frac{\omega}{c_{\mathrm{L}}}\right)^{2}-k^{2}}, \quad q=\sqrt{\left(\frac{\omega}{c_{\mathrm{T}}}\right)^{2}-k^{2}} .
$$

In the above expression, $\omega$ and $k$ are the angular frequency and the wave number of the Lamb mode, respectively, and $c_{\mathrm{L}}$ and $c_{\mathrm{T}}$ are the longitudinal and transverse wave speeds, respectively, of the elastic solid.

For a given angular frequency $\omega$, the displacement fields of the Lamb mode in the complex-value representation are given by ${ }^{17}$

$$
\begin{aligned}
& u_{1}\left(x_{1}, x_{3}, t\right)=\left(i k A \cos p x_{3}+q B \cos q x_{3}\right) \exp \left[i\left(k x_{1}-\omega t\right)\right], \\
& u_{3}\left(x_{1}, x_{3}, t\right)=\left(-p A \sin p x_{3}-i k B \sin q x_{3}\right) \exp \left[i\left(k x_{1}-\omega t\right)\right],
\end{aligned}
$$

for the $\mathrm{S}$ modes, where $A$ and $B$ are given as an eigenvector of

$$
\left[\begin{array}{ll}
-2 i k p \sin p h & \left(k^{2}-q^{2}\right) \sin q h \\
\left(k^{2}-q^{2}\right) \cos q h & -2 i k q \cos q h
\end{array}\right]\left(\begin{array}{l}
A \\
B
\end{array}\right)=\left(\begin{array}{l}
0 \\
0
\end{array}\right) .
$$

On the other hand, for the A modes,

$$
\begin{aligned}
& u_{1}\left(x_{1}, x_{3}, t\right)=\left(i k C \sin p x_{3}-q D \sin q x_{3}\right) \exp \left[i\left(k x_{1}-\omega t\right)\right], \\
& u_{3}\left(x_{1}, x_{3}, t\right)=\left(p C \cos p x_{3}-i k D \cos q x_{3}\right) \exp \left[i\left(k x_{1}-\omega t\right)\right],
\end{aligned}
$$

where $C$ and $D$ are given by

$$
\left[\begin{array}{ll}
2 i k p \cos q h & \left(k^{2}-q^{2}\right) \cos q h \\
\left(k^{2}-q^{2}\right) \sin p h & 2 i k q \sin q h
\end{array}\right]\left(\begin{array}{l}
C \\
D
\end{array}\right)=\left(\begin{array}{l}
0 \\
0
\end{array}\right) .
$$

In the above expressions, the wave motion is assumed to take place in the $x_{1} x_{3}$ plane with the propagation in the $x_{1}$ direction, as illustrated in Fig. 1.

For the following derivation, it is convenient to fix the phase velocity, $c_{\mathrm{p}}=\omega / k$, of the Lamb wave. Then, by substituting $k=\omega / c_{\mathrm{p}}$ in Eqs. (1) and (2), $\Omega_{\mathrm{S}}$ and $\Omega_{\mathrm{A}}$ can be regarded as functions of $\omega$. The problem to be discussed in

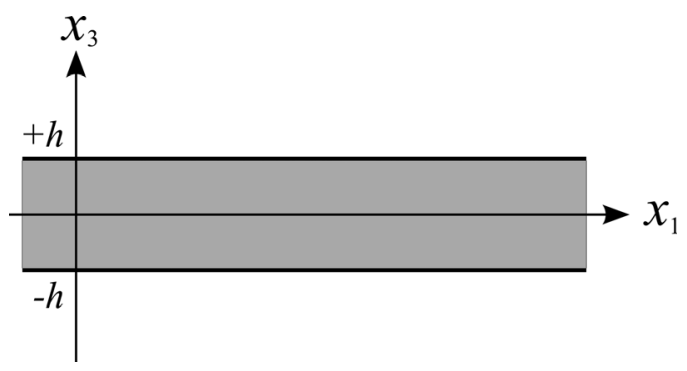

FIG. 1. Geometry of an elastic plate. 
this paper is to find a phase velocity $c_{\mathrm{p}}$ for which a set of equi-spaced angular frequencies $\left(\omega_{0}, 2 \omega_{0}, 3 \omega_{0}, \ldots\right)$ exists and each of its elements meets either Eq. (1) or Eq. (2), provided $\omega_{0}$ is real and positive. Once such a frequency set is found, an arbitrary element of the set can be chosen as the frequency of the primary Lamb mode, and its higher harmonics of any order have the same phase velocity.

\section{DERIVATION OF PHASE AND GROUP VELOCITY MATCHING}

In this section, the derivation of phase and group velocity matching is performed by exploiting periodicity properties of the trigonometric terms in Eqs. (1) and (2). It is useful here to note that the parameters $p$ and $q$ in Eq. (3) are both proportional to $\omega$. Below, the analysis is separately presented for three different regions of the phase velocity, namely, $c_{\mathrm{p}} \leq c_{\mathrm{T}}$ (Region I), $c_{\mathrm{T}}<c_{\mathrm{p}} \leq c_{\mathrm{L}}$ (Region II), and $c_{\mathrm{p}}>c_{\mathrm{L}}$ (Region III). It will be shown in Sec. IV that the derived conditions for the frequencies are not only sufficient but also necessary for the phase matching.

\section{A. Region I}

When $c_{\mathrm{p}} \leq c_{\mathrm{T}}$, the parameters $p$ and $q$ in Eq. (3) are both imaginary (including $q=0$ ), and it is convenient to rewrite Eqs. (1) and (2) with $\sin p h=i \sinh r h, \cos p h=\cosh r h$, sin $q h=i \sinh s h$ and $\cos q h=\cosh s h$, by defining real parameters $r$ and $s$ as $p=i r$ and $q=i s$. Each term in Eqs. (1) and (2) contains a product of a hyperbolic sine and a hyperbolic cosine. Since $r$ and $s$ are proportional to $\omega$, these trigonometric terms are not periodic in $\omega$. Therefore, it is not expected that there exists a series $\left(\omega_{0}, 2 \omega_{0}, 3 \omega_{0}, \ldots\right)$ which satisfies Eq. (1) or (2).

\section{B. Region II}

When $c_{\mathrm{T}}<c_{\mathrm{p}} \leq c_{\mathrm{L}}$, the parameter $p$ is imaginary (including $p=0$ ), while $q$ is real. In this circumstance, Eqs. (1) and (2) are rewritten with $\sin p h=i \sinh r h$ and $\cos p h=\cosh r h$ using $r$ as defined above. Noting that only $\sin q h$ and $\cos q h$ are periodic in $\omega$ in Eqs. (1) and (2), it is found that when one of the two terms of the left-hand side in each equation vanishes, the remaining term gives the desired series. In Eq. (1) for the $\mathrm{S}$ mode, this occurs when

$$
\left[q^{2}-\left(\frac{\omega}{c_{\mathrm{p}}}\right)^{2}\right]^{2}=0 \Leftrightarrow c_{\mathrm{p}}=\sqrt{2} c_{\mathrm{T}}, \quad q=\frac{\omega}{\sqrt{2} c_{\mathrm{T}}},
$$

or

$$
r=0 \Leftrightarrow c_{\mathrm{p}}=c_{\mathrm{L}}
$$

It is noted that Eq. (8) satisfies the condition $c_{\mathrm{T}}<c_{\mathrm{p}} \leq c_{\mathrm{L}}$ if Lamé's constant $\lambda$ is non-negative, for $c_{\mathrm{L}}^{2}-\left(\sqrt{2} c_{\mathrm{T}}\right)^{2}=\lambda$ / $\rho \geq 0$. For a somewhat pathological case of negative $\lambda$, the condition in Eq. (8) corresponds to the Region III to be discussed below, but the discussion goes parallel.
In Eq. (2) for the A mode, the corresponding condition is given by Eq. (8) only. The consequences of Eqs. (8) and (9) are examined in detail separately below.

\section{Case (II-1): $c_{\mathrm{p}}=\sqrt{\mathbf{2}} c_{\mathrm{T}}$}

In this case, the dispersion relations in Eqs. (1) and (2) reduce to

$S$ mode: $\quad \cos q h=0 . \quad \therefore q h=(2 n-1) \pi / 2$.

$$
\text { A mode: } \quad \sin q h=0 . \quad \therefore q h=n \pi,
$$

where $n$ is an integer. Accordingly, the angular frequency and the wave number are given by

$$
\begin{gathered}
\text { S mode: } \quad \omega=(2 n-1) \frac{\pi c_{\mathrm{T}}}{\sqrt{2} h}, k=(2 n-1) \frac{\pi}{2 h}, \\
\text { A mode: } \omega=2 n \frac{\pi c_{\mathrm{T}}}{\sqrt{2} h}, \quad k=2 n \frac{\pi}{2 h} .
\end{gathered}
$$

From Eqs. (12) and (13), it is seen that for this value of phase velocity, there exists a series of angular frequencies $\left[\left(N \pi c_{\mathrm{T}}\right) /\right.$ $(\sqrt{2} h) ; N=1,2,3, \ldots]$ satisfying the dispersion relation of $\mathrm{S}$ and $\mathrm{A}$ modes in an alternate manner.

The group velocity of the Lamb mode is obtained by

$$
\begin{aligned}
& \text { S mode: } \quad c_{\mathrm{g}}=-\frac{\partial \Omega_{\mathrm{S}} / \partial k}{\partial \Omega_{\mathrm{S}} / \partial \omega}, \\
& \text { A mode: } \quad c_{\mathrm{g}}=-\frac{\partial \Omega_{\mathrm{A}} / \partial k}{\partial \Omega_{\mathrm{A}} / \partial \omega},
\end{aligned}
$$

which yield for the above set of frequencies,

$$
c_{\mathrm{g}}=\frac{c_{\mathrm{T}}}{\sqrt{2}}
$$

irrespective of the mode and the integer $N$.

Therefore, the above set of frequencies gives the Lamb modes of not only an identical phase velocity but also of an identical group velocity. These Lamb modes are specially known as the Lamé modes (c.f. Graff ${ }^{17}$ ), which can be expressed as the superposition of bulk shear waves propagating in the directions with angles $\pm \pi / 4$ to the $x_{1}$-axis.

\section{Case (II-2): $c_{\mathrm{p}}=c_{\mathrm{L}}$}

When Eq. (9) holds, Eq. (1) reduces to

$$
\text { S mode: } \quad \sin q h=0 . \quad \therefore q h=n \pi,
$$

with $n$ being an integer, and $\omega$ and $k$ are given by

$$
\text { S mode: } \omega=\frac{n \pi}{h} \frac{c_{\mathrm{T}} c_{\mathrm{L}}}{\sqrt{c_{\mathrm{L}}^{2}-c_{\mathrm{T}}^{2}}}, \quad k=\frac{n \pi}{h} \frac{c_{\mathrm{T}}}{\sqrt{c_{\mathrm{L}}^{2}-c_{\mathrm{T}}^{2}}},
$$

which gives the desired series with the $\mathrm{S}$ modes alone. The corresponding group velocity can be obtained likewise as 
S mode: $\quad c_{\mathrm{g}}=\frac{c_{\mathrm{L}}\left[\left(c_{\mathrm{T}} / c_{\mathrm{L}}\right)^{2}+8 P\right]}{1+8 P}, P=\frac{1-\left(c_{\mathrm{T}} / c_{\mathrm{L}}\right)^{2}}{\left[2-\left(c_{\mathrm{L}} / c_{\mathrm{T}}\right)^{2}\right]^{2}}$,

again irrespective of $n$. In passing, it is noted that the condition obtained here corresponds to the symmetric Lamb modes with dominant longitudinal displacements as discussed by Pilarski et al. ${ }^{18}$ (see also Rose ${ }^{19}$ ).

\section{Region III}

Finally in this region with $c_{\mathrm{p}}>c_{\mathrm{L}}, p$ and $q$ are both real in Eqs. (1) and (2). In these equations, when one of $\sin p h$ and $\sin q h$ vanishes, the other necessarily vanishes, too. The same is true for cos $p h$ and $\cos q h$. Therefore, both terms on the left-hand side vanish together at certain values of $\omega$, when one of the following two conditions holds for each mode.

$$
\begin{aligned}
& \sin p h=\sin q h=0, \\
& \cos p h=\cos q h=0 .
\end{aligned}
$$

Moreover, when

$$
\left[q^{2}-\left(\frac{\omega}{c_{\mathrm{p}}}\right)^{2}\right]^{2}=4\left(\frac{\omega}{c_{\mathrm{p}}}\right)^{2} p q
$$

holds, the two terms on the left-hand side of both Eqs. (1) and (2) are combined to give

$$
\sin (p h+q h)=0 .
$$

The three cases with Eqs. (20), (21), and (23) are examined further below.

\section{Case (III-1): $\sin p h=\sin q h=0$}

In this case, $p$ and $q$ are given, using even integers $m$ and $n$, as

$$
p h=\frac{1}{2} m \pi, q h=\frac{1}{2} n \pi,
$$

where $m<n$ since $c_{\mathrm{T}}<c_{\mathrm{L}}$ implies $p<q$. From Eq. (24), the phase velocity is, for both modes, given by

$$
c_{\mathrm{p}}=c_{\mathrm{T}} \sqrt{\frac{1-\eta^{2}}{\zeta^{2}-\eta^{2}}}
$$

where $\eta=m / n, \zeta=c_{\mathrm{T}} / c_{\mathrm{L}}$. In order for the phase velocity to be real, the ratio $\eta$ should be smaller than $\zeta$, so $\eta$ is restricted in the range $\eta<\zeta<1$.

Substituting Eq. (25) into Eq. (3) and further into Eq. (24), $\omega$ and $k$ are given by

$$
\omega=\frac{n \pi c_{\mathrm{T}}}{2 h} \sqrt{\frac{1-\eta^{2}}{1-\zeta^{2}}}, k=\frac{n \pi}{2 h} \sqrt{\frac{\zeta^{2}-\eta^{2}}{1-\zeta^{2}}},
$$

with $n$ being an even integer, which gives a desired series. It is noted here that the phase velocity of Eq. (25), and each angular frequency given by Eq. (26), satisfy the dispersion relation for the $S$ and A modes, Eqs. (1) and (2), simultaneously. This means that these frequencies and phase velocities give intersecting points of the dispersion curves of $\mathrm{S}$ and $\mathrm{A}$ modes. At these frequencies, however, the corresponding group velocities differ depending on the mode symmetry, and given by Eqs. (14) and (15) as

S mode: $\quad c_{\mathrm{g}}=\frac{\left(1-\eta^{2}\right)^{2}}{\left(1-\eta^{2}\right)^{2}-4\left(\zeta^{2}-\eta^{2}\right)\left(1-\zeta^{2}\right)^{2}} \sqrt{\frac{\zeta^{2}-\eta^{2}}{1-\eta^{2}}} c_{\mathrm{T}}$,

$$
\text { A mode: } \begin{aligned}
c_{\mathrm{g}}= & \frac{\left(1-2 \zeta^{2}+\eta^{2}\right)^{2}+4 \eta^{2}\left(\zeta^{2}-\eta^{2}\right)\left(1-\zeta^{2}\right)}{\zeta^{2}\left(1-2 \zeta^{2}+\eta^{2}\right)^{2}+4 \eta^{2}\left(\zeta^{2}-\eta^{2}\right)\left(1-\zeta^{2}\right)} \\
& \times \sqrt{\frac{\zeta^{2}-\eta^{2}}{1-\eta^{2}}} c_{\mathrm{T}} .
\end{aligned}
$$

\section{Case (III-2): $\cos p h=\cos q h=0$}

In this case, $p$ and $q$ are given by

$$
p h=\frac{1}{2} m \pi, q h=\frac{1}{2} n \pi,
$$

using odd integers $m$ and $n$, where again $m<n$. Equation (29) gives the phase velocity in the same form as Eq. (25), while Eq. (26) applies here for $\omega$ and $k$, but with $n$ being an odd integer. The corresponding group velocities are identical to Eqs. (27) and (28), respectively, where the correspondence to the mode symmetry is interchanged, namely, Eq. (28) for the S mode, and Eq. (27) for the A mode.

The set of angular frequencies in this case does not conform to the desired form, since the entries of the series in Eq. (26) are proportional to odd integers only. When supplemented by the one derived in (III-1), however, it gives a sought-for series. In fact, for an arbitrary $\eta=m / n$ with $m$ and $n$ both being odd integers, one can always find a pair of even integers $2 m$ and $2 n$ to give the same ratio $\eta$. Therefore, the above series can be combined with the series of (III-1) to make the following desired form

$$
\left[\frac{\pi c_{\mathrm{T}}}{2 h} \sqrt{\frac{1-\eta^{2}}{1-\zeta^{2}}}, \frac{2 \pi c_{\mathrm{T}}}{2 h} \sqrt{\frac{1-\eta^{2}}{1-\zeta^{2}}}, \frac{3 \pi c_{\mathrm{T}}}{2 h} \sqrt{\frac{1-\eta^{2}}{1-\zeta^{2}}}, \cdots \cdots\right],
$$

whose elements meet the dispersion relations with the same phase velocity of Eq. (25).

\section{Case (III-3): $\sin (p h+q h)=0$}

In this case, both Eqs. (1) and (2) lead to

$$
p h+q h=n \pi,
$$

where $n$ is an integer, and this gives the desired equi-spaced frequencies once the value of $c_{\mathrm{p}}$ is established. 
By putting $x=c_{\mathrm{p}} / c_{\mathrm{T}}$ and $\zeta=c_{\mathrm{T}} / c_{\mathrm{L}}$, Eq. (22) reduces to

$$
x^{6}-8 x^{4}+8\left(3-2 \zeta^{2}\right) x^{2}+16\left(\zeta^{2}-1\right)=0,
$$

which is identical to the characteristic equation for the Rayleigh wave. This equation has three roots for $x^{2}$, one of which always gives a real velocity, corresponding to the wellknown Rayleigh wave on the free surface of an isotropic solid. The other two roots give real-valued velocities only when

$$
11-62 \zeta^{2}+107 \zeta^{4}-64 \zeta^{6}<0 \Leftrightarrow \zeta>\zeta_{\mathrm{cr}} \equiv 0.5670 \ldots \ldots
$$

is satisfied. Provided $\zeta$ satisfies this inequality, the three phase velocities can be expressed as

$$
\begin{gathered}
c_{\mathrm{R} 1}, c_{\mathrm{R} 2}=c_{\mathrm{T}} \sqrt{\frac{8}{3}+\frac{4}{3}\left(\frac{-Q \pm \sqrt{3} R}{2}\right)}, \\
c_{\mathrm{R} 3}=c_{\mathrm{T}} \sqrt{\frac{8}{3}+\frac{4}{3} Q},
\end{gathered}
$$

where the plus $(+)$ and minus $(-)$ signs in Eq. (34) correspond to $c_{\mathrm{R} 1}$ and $c_{\mathrm{R} 2}$, respectively, and $Q$ and $R$ are real constants given by

$$
\begin{aligned}
& {\left[-17+45 \zeta^{2}+3 \sqrt{3} \sqrt{-\left(11-62 \zeta^{2}+107 \zeta^{4}-64 \zeta^{6}\right)} i\right]^{1 / 3}} \\
& =Q+i R .
\end{aligned}
$$

It is noted that there exist three choices for $Q$ and $R$, as the left-hand side of Eq. (36) has three cubic roots in the complex domain. Different choices of $Q$ and $R$, however, merely interchange the phase velocities among the values of Eqs. (34) and (35). Without loss of generality, then, it can be assumed that Eq. (35) corresponds to the velocity of the Rayleigh surface wave.

With this choice of $Q$ and $R$, the angular frequencies for $c_{\mathrm{R} 1}$ and $c_{\mathrm{R} 2}$ are given by Eq. (34) as

$$
\begin{aligned}
\omega_{\mathrm{R} 1}, \omega_{\mathrm{R} 2}= & n \frac{\pi c_{\mathrm{T}}}{h} \\
& \times \frac{\sqrt{8-2 Q \pm 2 \sqrt{3} R}}{\sqrt{5-2 Q \pm 2 \sqrt{3} R}+\sqrt{\zeta^{2}(8-2 Q \pm 2 \sqrt{3} R)-3}},
\end{aligned}
$$

respectively ( $n$ : integer). Equation (37) gives the desired equi-spaced frequencies on the dispersion curves with common phase velocities. It is noted that these frequencies and the corresponding phase velocity are on the intersections of the $\mathrm{A}$ and $\mathrm{S}$ modes. For these frequencies, however, Eqs. (14) and (15) do not yield a common group velocity.

For the Rayleigh surface wave, it is well known that $c_{\mathrm{R} 3}$ is less than $c_{\mathrm{T}}$. Therefore, from Eq. (3), no real-valued frequency exists on the dispersion curves for this phase velocity. It has been already concluded in the discussion for the Region I that there is no set of equi-spaced frequencies that satisfy the Rayleigh-Lamb frequency equation for a common phase velocity. It is noted in passing, however, that Müller et $a l .{ }^{16}$ classified this case as the phase matching in an asymptotic sense in the high-frequency limit.

\section{NECESSITY OF THE DERIVED CONDITIONS}

In the previous section, some phase velocities have been derived for which the Rayleigh-Lamb frequency equations are satisfied at equi-spaced frequencies. In this Section, it is shown that no other such phase velocities exist, namely, that the possible phase velocities have been exclusively discussed already. To this purpose, it suffices to show that the conditions for the phase velocity derived above are necessary for a primary and its second-harmonic modes to have the same phase velocity.

For convenience, the discussion is started with the Region III. Depending on the mode symmetry of the primary and the second-harmonic modes, the following four cases need to be considered separately, namely, the second-harmonic generation of (a) an $\mathrm{S}$ mode into an $\mathrm{S}$ mode, (b) an $\mathrm{A}$ mode into an A mode, (c) an S mode into an A mode and (d) an $\mathrm{A}$ mode into an $\mathrm{S}$ mode (although the aspect of nonzero power flow ${ }^{9,13}$ may preclude the second-harmonic generation into an A mode, the attention is paid to all formally possible cases here). Since these four cases can be examined in a similar manner, only the case for (a) is described here because of the limited space.

The conditions for the case (a), namely, that a primary $S$ mode and its second-harmonic $S$ mode have the same phase velocity, are that Eq. (1) are satisfied for $\Omega_{\mathrm{S}}(\omega, k)=0$ and $\Omega_{\mathrm{S}}(2 \omega, 2 k)=0$, namely,

$$
\begin{aligned}
K_{1} \sin q h \cos p h+K_{2} \sin p h \cos q h & =0, \\
K_{1} \sin 2 q h \cos 2 p h+K_{2} \sin 2 p h \cos 2 q h & =0,
\end{aligned}
$$

where the coefficients are simply put as $K_{1}=\left(q^{2}-k^{2}\right)^{2}$, $K_{2}=4 k^{2} p q$. Subtracting Eq. (39) multiplied by $\sin p h \cos q h$ from Eq. (38) multiplied by $\sin 2 p h \cos 2 q h$, one obtains

$$
K_{1} \sin p h \sin q h(\cos p h-\cos q h)(\cos p h+\cos q h)=0,
$$

after some manipulation. Since $K_{1}>0$ in the Region III, Eq. (40) requires (i) $\sin p h=0$, (ii) $\sin q h=0$, (iii) $\cos p h$ - $\cos q h=0$ or (iv) $\cos p h+\cos q h=0$. Clearly from Eq. (38), (i) and (ii) are equivalent and each leads to the case (III-1) in Sec. III. On the other hand, both (iii) and (iv) imply $\sin p h= \pm \sin q h$, which yields (III-1), (III-2) or $K_{1}=K_{2}$ from Eq. (38) as $K_{1}$ and $K_{2}$ are both positive. The third case has been already discussed in the case (III-3). For the case (b), similar reasoning as above leads to Eq. (40), so this situation also corresponds to (III-1)-(III-3) already discussed. Likewise, in the cases (c) and (d), the same three cases are recovered.

The above discussion has proved that the phase matching of a primary and its second harmonic modes in the Region III only occurs for the special values of the phase velocity derived in Sec. III. 
Next, for the Region II, one can also examine the four cases (a)-(d) of the second-harmonic generation to show that the conditions derived in Sec. III cover all possibilities for the phase matching, namely, the cases of (II-1) and (II-2) are obtained as the necessary conditions.

For the Region I, a similar manipulation results in the expressions involving only hyperbolic functions, so the phase matching is not possible.

Obviously, the necessary conditions of the phase matching for the second harmonic generation obtained here are also necessary for the harmonic generation of arbitrary order. As a result, the conditions for the phase velocity derived in Sec. III cover all possible cases of the phase matching occurring in Lamb waves for higher harmonics of arbitrary order.

\section{DISCUSSION}

\section{A. Summary of phase and group velocity matching}

The results derived in Sec. III can be summarized as the following four mode types. Furthermore, for each type, the connection of the order of each element in the frequency set to the order of the Lamb mode is established below.

\section{Type (i): Lamé modes}

For the phase velocity $c_{\mathrm{p}}=\sqrt{2} c_{\mathrm{T}}$, the frequency set is given by Eqs. (12) and (13), which corresponds, in terms of the frequency-thickness product, to

$$
f d=\frac{N c_{\mathrm{T}}}{\sqrt{2}}, \quad N=1,2, \ldots \ldots
$$

The group velocity is given by Eq. (16). The frequencies ordered as above give an $\mathrm{S}$ mode and an A mode in an alternate manner, i.e., $S_{0}, A_{1}, S_{1}, A_{2}$, and so on. In a general notation, $N$ in Eq. (41) is associated to the $k$ th-order symmetric mode when $N$ is odd and $k=(N-1) / 2$, and to the $k$ th-order antisymmetric mode when $N$ is even and $k=N / 2$.

\section{Type (ii): Symmetric modes with dominant longitudinal displacements}

For the phase velocity $c_{\mathrm{p}}=c_{\mathrm{L}}$, the frequency set is given by Eq. (18), yielding

$$
f d=\frac{N c_{\mathrm{T}} c_{\mathrm{L}}}{\sqrt{c_{\mathrm{L}}^{2}-c_{\mathrm{T}}^{2}}}, N=1,2, \ldots \ldots
$$

The group velocity is given by Eq. (19). The frequencies of this set are all related to the $\mathrm{S}$ mode, and $N$ in Eq. (42) corresponds to the $N$ th-order symmetric mode. This type has been previously identified by Müller et al. ${ }^{16}$ in the special case of second-harmonic generation. Here, it is shown to characterize the harmonic generation of arbitrary order.

\section{Type (iii): Intersections of symmetric and antisymmetric modes}

The phase velocity $c_{\mathrm{p}}=c_{\mathrm{T}}\left[\left(1-\eta^{2}\right) /\left(\zeta^{2}-\eta^{2}\right)\right]^{1 / 2}$ in Eq. (25) is characterized by the ratio $\eta$ of two integers $m$ and $n$, under the condition of $\eta=m / n<\zeta=c_{\mathrm{T}} / c_{\mathrm{L}}$. The angular frequency sets are given by Eq. (26), which applies whether $n$ is odd or even. In the case of second-harmonic generation, this type has also been discussed by Müller et al. ${ }^{16}$ In order to demonstrate the structure of the frequency sets for arbitrary harmonics, this case is now re-categorized into the following two types, depending on whether the frequency set consists of frequencies given by (III-1) and (III-2) jointly or the frequencies given by (III-1) only.

a. Type (iii-a): When $m$ and $n$ are both odd integers and $\eta=m / n$, the same $\eta$ can be given as the ratio of even integers, i.e., $2 m / 2 n$. Then, denoting the smallest pair of odd integers giving this ratio $\eta$ by $m_{0}$ and $n_{0}$, Eq. (26) and the corresponding frequency-thickness products can be expressed in a unified manner as

$$
\begin{aligned}
\omega & =\frac{N n_{0} \pi c_{\mathrm{T}}}{2 h} \sqrt{\frac{1-\eta^{2}}{1-\zeta^{2}}}, \\
f d & =\frac{N n_{0} c_{\mathrm{T}}}{2} \sqrt{\frac{1-\eta^{2}}{1-\zeta^{2}}}, \quad N=1,2, \ldots \ldots
\end{aligned}
$$

where $\eta=m_{0} / n_{0}=m / n$ and $N=n / n_{0}=m / m_{0}$, combining the two cases (III-1) and (III-2) discussed above. These frequencies are associated with the same phase velocity, but with two group velocities, namely, Eq. (28) for S modes with odd $N$ and for A modes with even $N$, and Eq. (27) for A modes with odd $N$ and for $\mathrm{S}$ modes with even $N$. Each group velocity is shared by $\mathrm{A}$ and $\mathrm{S}$ modes in an alternate order.

The above summary obviously includes the case when $m$ and $n$ are both even and their ratio $\eta=m / n$ can be also given as the ratio of two odd integers. The remaining case is described below.

b. Type (iii-b): When $m$ and $n$ are both even integers but their ratio $\eta=m / n$ cannot be given as the ratio of two odd integers, the same expression in Eq. (43) gives the desired frequency set, using the smallest pair of even integers $m_{0}$ and $n_{0}$ giving this ratio $\eta$. The frequencies are again related to the above phase velocity but two different group velocities as Eqs. (27) and (28) corresponding to S and A modes, respectively. Each group velocity is separately linked to $\mathrm{A}$ or $\mathrm{S}$ mode.

The order of the pertinent Lamb mode for the type (iii) can be naturally identified through the careful analysis of the number of roots of Eqs. (1) and (2) along a straight line $\omega=c_{\mathrm{p}} k$. After some analysis, it reveals that the Lamb mode characterized by two integers $m$ and $n$ in the mode type (iii) corresponds to the $M$ th-order symmetric or antisymmetric mode, where $M=(m+n) / 2$.

\section{Type (iv): Extra Rayleigh modes}

The phase velocities in Eq. (34) have been derived as the two extraneous roots of the characteristic equation for the Rayleigh wave. Hereafter, the Lamb modes with these phase velocities are referred to as the extra Rayleigh modes. Importantly, this mode type is possible only when $c_{\mathrm{T}} / c_{\mathrm{L}}$ is 
greater than $\zeta_{\mathrm{cr}}$ defined in Eq. (33). From Eq. (37), the frequency-thickness products for this type are given by

$f d=N c_{\mathrm{T}} \frac{\sqrt{8-2 Q \pm 2 \sqrt{3} R}}{\sqrt{5-2 Q \pm 2 \sqrt{3} R}+\sqrt{\zeta^{2}(8-2 Q \pm 2 \sqrt{3} R)-3}}$,

$N=1,2, \ldots \ldots$

with plus and minus signs for $c_{\mathrm{p}}=c_{\mathrm{R} 1}$ and $c_{\mathrm{p}}=c_{\mathrm{R} 2}$, respectively. The phase velocities and the corresponding frequencies lie on the intersecting points of symmetric and antisymmetric modes, but this Lamb mode type is deliberately separated from the mode type (iii) above, since this type has some distinguished properties. For instance, the matching of the group velocity cannot be established for this type. By the argument similar to the one used for the previous type (iii), it can be seen that Eq. (44) corresponds to the $N$ th-order symmetric and antisymmetric modes.

\section{B. Surface motions of Lamb modes with phase matching}

Each of the four mode types as summarized above is shown to have characteristic surface motions. These are clarified by examining the through-thickness distribution of the displacement components given by Eqs. (4) and (6).

\section{Type (i): Lamé modes}

Substituting Eq. (8) into Eq. (5) gives $A=0$, which together with Eqs. (4) and (10) gives $u_{1}\left(x_{1}, \pm h, t\right)=0$ for the $\mathrm{S}$ modes. Likewise, Eqs. (6)-(8) and (11) give $C=0$ and $u_{1}\left(x_{1}, \pm h, t\right)=0$ for the A modes. Therefore, these Lamb modes consist of the $\mathrm{S}$ and A modes with vanishing horizontal displacements on both surfaces of the plate.

\section{Type (ii): Symmetric modes with dominant longitudinal displacements}

From Eqs. (4), (5), (9) and (17), the surface motion satisfies $u_{3}\left(x_{1}, \pm h, t\right)=0$, demonstrating that the vertical displacement on both surfaces vanish for this mode type.

\section{Type (iii): Intersections of symmetric and antisymmetric modes}

For this mode type, the surface motions are determined by Eqs. (4)-(7), (20), and (21), and the parity of $m$ and $n$. For the $\mathrm{S}$ modes, $u_{3}\left(x_{1}, \pm h, t\right)=0$ when $m$ and $n$ are both even, and $u_{1}\left(x_{1}, \pm h, t\right)=0$ when $m$ and $n$ are both odd. On the other hand, for the A modes, $u_{1}\left(x_{1}, \pm h, t\right)=0$ when $m$ and $n$ are both even, and $u_{3}\left(x_{1}, \pm h, t\right)=0$ when $m$ and $n$ are both odd. Therefore, the Lamb modes considered here are those either with vanishing vertical or horizontal displacement on both surfaces of the plate, depending on the mode symmetry and the parity of the integers $m$ and $n$.

\section{Type (iv): Extra Rayleigh modes}

From Eqs. (4)-(7), (31) and (34), the displacements for this type are given by

$$
\begin{aligned}
u_{1}= & i K \sqrt{q}\left[\left(\sqrt{\frac{N \pi}{p h}}-1\right) \cos p x_{3}+(-1)^{N} \cos q x_{3}\right] \\
& \times \exp \left[i\left(k x_{1}-\omega t\right)\right], \\
u_{3}= & K \sqrt{p}\left[-\sin p x_{3}+(-1)^{N}\left(\sqrt{\frac{N \pi}{p h}}-1\right) \sin q x_{3}\right] \\
& \times \exp \left[i\left(k x_{1}-\omega t\right)\right],
\end{aligned}
$$

for the $\mathrm{S}$ modes, and

$$
\begin{aligned}
u_{1}= & i K \sqrt{q}\left[\left(\sqrt{\frac{N \pi}{p h}}-1\right) \sin p x_{3}-(-1)^{N} \sin q x_{3}\right] \\
& \times \exp \left[i\left(k x_{1}-\omega t\right)\right], \\
u_{3}= & K \sqrt{p}\left[\cos p x_{3}+(-1)^{N}\left(\sqrt{\frac{N \pi}{p h}}-1\right) \cos q x_{3}\right] \\
& \times \exp \left[i\left(k x_{1}-\omega t\right)\right],
\end{aligned}
$$

for the A modes. The parameters $p$ and $q$ in the above expressions are given in terms of $Q$ and $R$ defined in Eq. (36) by

$$
\begin{aligned}
& p=\frac{N \pi}{h}\left(1+\sqrt{\frac{2(4-Q \pm \sqrt{3} R)-3}{2 \zeta^{2}(4-Q \pm \sqrt{3} R)-3}}\right)^{-1}, \\
& q=\frac{N \pi}{h}\left(1+\sqrt{\frac{2 \zeta^{2}(4-Q \pm \sqrt{3} R)-3}{2(4-Q \pm \sqrt{3} R)-3}}\right)^{-1},
\end{aligned}
$$

with the plus and minus signs corresponding to $c_{\mathrm{p}}=c_{\mathrm{R} 1}$ and $c_{\mathrm{p}}=c_{\mathrm{R} 2}$, respectively. The surface motion for this mode type is therefore not horizontal or vertical, but it is featured with an elliptic trajectory.

To summarize, in the first three mode types (i)-(iii) among the four given above, the Lamb modes exhibit the phase and group velocity matching and have special features of their surface motion. Namely, they are characterized either by vanishing vertical displacement or vanishing horizontal displacement on the traction-free surfaces. On the other hand, the mode type (iv) involves an elliptic particle motion of the plate surfaces. In the case of second-harmonic generation, similar features have been noted by Müller et al. ${ }^{16}$ for the types (ii) and (iii).

These features may have certain significant implications to the excitation of the primary Lamb mode and detection of its harmonics in practical nonlinear ultrasonic measurements. For example, the type (ii) is featured by purely horizontal surface motions, so it is expected to be difficult to excite or detect the pertinent Lamb modes with immersion methods or by angle-beam transducers connected with liquid couplant. The types (i) and (iv) are suitable for this purpose as the relevant Lamb modes have vertical horizontal motions. For the type (iii), this issue depends on the particular values of $m$ and $n$ as well as the mode symmetry. 


\section{Additional remarks}

In the foregoing studies, some specific frequencies of the primary Lamb mode that exhibit the phase matching have been explicitly discussed. These are examined here in the light of the conditions derived here.

Deng $^{7}$ obtained an existence condition for cumulative generation of the second harmonics as

$$
\tan p h=\tan q h \Leftrightarrow q h-p h=k \pi,
$$

in the notation employed here, where $k$ is an integer. This expression can be obtained from Eq. (24) or Eq. (29) in the present analysis. Our results in Eqs. (24) and (29), however, specify the possible frequencies more restrictively. Moreover, the present discussion has revealed another condition $q h+p h=k \pi$ for the phase matching (extra Rayleigh modes). In fact, the crossing points corresponding to this condition can be found in Deng's plot (Fig. 4 in Ref. 7), with no particular mention though. In Sec. VI, this mode type is demonstrated using the material constants from Ref. 7.

By graphical and numerical arguments, Deng recorded some explicit values as $\left(k h, c_{\mathrm{p}}\right)=(2.57,5.47 \mathrm{~km} / \mathrm{s})$ for an $\mathrm{S}$ mode for an iron plate. This corresponds to the type (iii), the $\mathrm{S}$ modes with dominant longitudinal displacements, with $\mathrm{kh}$ given in Eq. (18). Deng also describes $(3.01,6.12 \mathrm{~km} / \mathrm{s})$ for an $\mathrm{S}$ mode and $(3.00,6.14 \mathrm{~km} / \mathrm{s}),(2.72,8.32 \mathrm{~km} / \mathrm{s})$ for $\mathrm{A}$ modes. These values can be readily obtained from our results for the type (iii), the intersections of $\mathrm{S}$ and $\mathrm{A}$ modes. Namely, the first two cases correspond to $(m, n)=(1,3)$ and the third case to $(2,4)$, with $c_{\mathrm{p}}$ given in Eq. (25) and $k$ in Eq. (26).

Deng ${ }^{8}$ later discussed the case of the cumulative second harmonic generation from the primary $\mathrm{A}_{4}$ and $\mathrm{S}_{4}$ modes at $f h$ $(=f d / 2)=5.03 \mathrm{MHz} \cdot \mathrm{mm}$ and $c_{\mathrm{p}}=8.206 \mathrm{~km} / \mathrm{s}$ for an aluminum plate. This is also a special case of the type (iii) with $(m, n)=(2,6)$. Deng et al. ${ }^{10}$ attempted an experimental verification of cumulative growth of second harmonics in Lamb waves, where they used the $\mathrm{A}_{2}$ and $\mathrm{S}_{2}$ modes at $2.70 \mathrm{MHz}$, $c_{\mathrm{p}}=8.194 \mathrm{~km} / \mathrm{s}$, for a $1.85 \mathrm{~mm}$-thick aluminum plate. This also corresponds to the case $(m, n)=(1,3)$ in Eq. (25) (with odd $n$ ) and in Eq. (42).

Bermes et al. ${ }^{11}$ experimentally used the $2.15 \mathrm{MHz} \mathrm{S}_{1}$ mode for a $1.6 \mathrm{~mm}$-thick aluminum plate and observed the second harmonic generation into $\mathrm{S}_{2}$ mode. Likewise, Pruell et al. $^{12}$ measured the second harmonic generation from the 2.225 $\mathrm{MHz}_{1}$ mode into $\mathrm{S}_{2}$ mode. These examples correspond to the symmetric modes with dominant longitudinal displacements of Eq. (42), with $N=1$ for the primary mode. It is noted that just recently, Matlack et al. ${ }^{20}$ provided some quantitative experimental results for the second harmonic generation corresponding to this mode type, i.e., $S_{1}$ into $S_{2}$, and $\mathrm{S}_{2}$ into $\mathrm{S}_{4}$ modes.

In an experimental study, Lee et al. ${ }^{14}$ used the $\mathrm{A}_{1}$ primary mode at $2.2 \mathrm{MHz}$ for a $2 \mathrm{~mm}$-thick aluminum plate and recorded the second harmonic generation in the $A_{2}$ mode. This case corresponds to the Lamé mode with the primary mode given by Eq. (41), when $N=1$. It is noted, however, that the occurrence of the second harmonic generation into antisymmetric Lamb modes is theoretically precluded according to the analysis of Deng ${ }^{7,8}$ (see, also Srivastava and Lanza di Scalea ${ }^{13}$ ).

Srivastava and Lanza di Scalea ${ }^{13}$ also made measurements for higher harmonic generation in Lamb waves. For a $2.54 \mathrm{~mm}$-thick aluminum plate, they used low-frequency symmetric as well as antisymmetric modes at $0.32 \mathrm{MHz}$ as the input wave. These cases are, however, not of a cumulative nature according to the results of the present discussion.

As mentioned in the Introduction, Müller et al. ${ }^{16}$ obtained five mode types which satisfy the phase and group velocity matching between the primary and its second harmonic Lamb modes. Namely, they are (C) crossing points of symmetric and antisymmetric modes, (L) symmetric modes with longitudinal phase velocity, $(\mathrm{O})$ nonzero order modes near cut-off frequencies, (T) nonzero order modes at high wave numbers, and $(\mathrm{R})$ the lowest-order modes at high wave numbers (quasi-Rayleigh wave in their terminology). Among them, the mode types (C) and (L) correspond to the mode types (iii) and (ii), respectively, in the present formulation. The counterparts of the mode types (i) and (iv) derived here are not mentioned by Müller et al. ${ }^{16}$ Their mode types (O), (T) and (R) are of asymptotic nature, where Lamb modes attain either a cut-off or a nondispersive behavior. Such approximate matching conditions may be of some practical use, but these are not discussed here.

Foregoing investigators also examined the nonzero power transfer from the primary to the harmonic modes as a necessary condition for their cumulative growth. For the second harmonics, the previous studies ${ }^{7,8,13,16}$ have shown by symmetry arguments that a symmetric mode may be cumulatively generated while an antisymmetric mode is excluded, irrespective of the symmetry of the primary Lamb mode. Considerations for the harmonic generation of other orders are given by Srivastava and Lanza di Scalia. ${ }^{13}$ Such requirements certainly restrict the possible mode and frequency among the mode types obtained in this paper for cumulative harmonic generation. For example, the Lamé modes in the type (i) always have A modes at even orders, so for any primary mode the second harmonic generation is impossible in this sense. This fact, however, does not reduce the significance of the Lamé modes, since they encompass e.g. the third-harmonic generation in A modes due to the cubic nonlinearity.

\section{GRAPHICAL DEMONSTRATIONS}

Finally, the modes and the frequencies of Lamb waves which meet the conditions for the phase and group velocity matching are explicitly demonstrated for specific materials.

For an aluminum plate, for which $c_{\mathrm{L}}=6350 \mathrm{~m} / \mathrm{s}$ and $c_{\mathrm{T}}=3130 \mathrm{~m} / \mathrm{s}$, Table I illustrates the three types derived and summarized in Sec. V. Note that the type (iv), extra Rayleigh modes, is not existent as $c_{\mathrm{T}} / c_{\mathrm{L}}=0.49 \ldots \ldots<\zeta_{\mathrm{cr}}$. The mode types (i) and (ii) are characterized by a single frequency set and a single phase velocity for each. On the other hand, the mode type (iii) involves infinite numbers of the phase velocity by different combinations of the integers $m_{0}$ and $n_{0}$, so only the cases for which the first entry is the Lamb mode of 
TABLE I. Mode types exhibiting the phase and group velocity matching for an aluminum plate $\left([f d]_{=1}\right.$ denotes the smallest element of the matching frequency-thickness set)

\begin{tabular}{|c|c|c|c|c|}
\hline \multicolumn{2}{|c|}{ Lamb modes } & $\begin{array}{l}{[f d]_{N=1}} \\
(\mathrm{MHz}: \mathrm{mm})\end{array}$ & $\begin{array}{l}\text { Phase velocity } \\
(\mathrm{km} / \mathrm{s})\end{array}$ & $\begin{array}{l}\text { Group veloc } \\
(\mathrm{km} / \mathrm{s})\end{array}$ \\
\hline \multicolumn{5}{|c|}{ (i) Lamé modes } \\
\hline \multicolumn{2}{|c|}{$\mathrm{S}_{0}-\mathrm{A}_{1}-\mathrm{S}_{1}-\ldots \ldots$} & 2.213 & 4.426 & 2.213 \\
\hline \multicolumn{5}{|c|}{ (ii) Symmetric modes with dominant longitudinal displacements } \\
\hline \multicolumn{2}{|c|}{$\mathrm{S}_{1}-\mathrm{S}_{2}-\mathrm{S}_{3}-\ldots \ldots$} & 3.597 & 6.35 & 4.307 \\
\hline \multicolumn{5}{|c|}{$\begin{array}{l}\text { (iii) Intersections of symmetric and antisymmetric modes } \\
\left(m_{0}, n_{0}\right)\end{array}$} \\
\hline$(1,3)$ & $\begin{array}{l}\mathrm{S}_{2}-\mathrm{A}_{4}-\mathrm{S}_{6}-\ldots \ldots \\
\mathrm{A}_{2}-\mathrm{S}_{4}-\mathrm{A}_{6}-\ldots \ldots\end{array}$ & 5.088 & 8.127 & $\begin{array}{l}3.766 \\
1.952\end{array}$ \\
\hline$(1,5)$ & $\begin{array}{l}\mathrm{S}_{3}-\mathrm{A}_{6}-\mathrm{S}_{9}-\ldots \ldots \\
\mathrm{A}_{3}-\mathrm{S}_{6}-\mathrm{A}_{9}-\ldots \ldots\end{array}$ & 8.812 & 6.807 & $\begin{array}{l}4.812 \\
2.907\end{array}$ \\
\hline$(1,7)$ & $\begin{array}{l}\mathrm{S}_{4}-\mathrm{A}_{8}-\mathrm{S}_{12}-\ldots \ldots \\
\mathrm{A}_{4}-\mathrm{S}_{8}-\mathrm{A}_{12}-\ldots \ldots\end{array}$ & 12.46 & 6.567 & $\begin{array}{l}5.372 \\
3.186\end{array}$ \\
\hline$(1,9)$ & $\begin{array}{l}\mathrm{S}_{5}-\mathrm{A}_{10}-\mathrm{S}_{15}-\ldots \ldots \\
\mathrm{A}_{5}-\mathrm{S}_{10}-\mathrm{A}_{15}-\ldots \ldots\end{array}$ & 16.09 & 6.477 & $\begin{array}{l}5.690 \\
3.302\end{array}$ \\
\hline$(2,8)$ & $\begin{array}{l}\mathrm{A}_{5}-\mathrm{A}_{10}-\mathrm{A}_{15^{-}} \ldots \ldots \\
\mathrm{S}_{5}-\mathrm{S}_{10}-\mathrm{S}_{15}-\ldots \ldots\end{array}$ & 13.93 & 7.134 & $\begin{array}{l}4.380 \\
2.594\end{array}$ \\
\hline$(3,7)$ & $\begin{array}{l}\mathrm{S}_{5}-\mathrm{A}_{10}-\mathrm{S}_{15}-\ldots \ldots \\
\mathrm{A}_{5}-\mathrm{S}_{10}-\mathrm{A}_{15}-\ldots \ldots\end{array}$ & 11.38 & 11.61 & $\begin{array}{l}2.899 \\
1.060\end{array}$ \\
\hline$(1,11)$ & $\begin{array}{l}\mathrm{S}_{6}-\mathrm{A}_{12}-\mathrm{S}_{18^{-}} \ldots \ldots \\
\mathrm{A}_{6}-\mathrm{S}_{12}-\mathrm{A}_{18^{-}} \ldots \ldots\end{array}$ & 19.70 & 6.434 & $\begin{array}{l}5.881 \\
3.362\end{array}$ \\
\hline
\end{tabular}

(iv) Extra Rayleigh modes: non-existent $(\zeta=0.49 \ldots<0.567)$

TABLE II. Mode types exhibiting the phase and group velocity matching for an iron plate $\left([f d]_{N=1}\right.$ denotes the smallest element of the matching frequency-thickness set).

\begin{tabular}{|c|c|c|c|c|}
\hline \multicolumn{2}{|c|}{ Lamb modes } & $\begin{array}{c}{[f d]_{N=1}} \\
(\mathrm{MHz} \cdot \mathrm{mm})\end{array}$ & $\begin{array}{l}\text { Phase velocity } \\
\quad(\mathrm{km} / \mathrm{s})\end{array}$ & $\begin{array}{l}\text { Group velocity } \\
(\mathrm{km} / \mathrm{s})\end{array}$ \\
\hline \multicolumn{5}{|c|}{ (i) Lamé modes } \\
\hline \multicolumn{2}{|c|}{$\mathrm{S}_{0}-\mathrm{A}_{1}-\mathrm{S}_{1}-\ldots \ldots$} & 2.336 & 4.671 & 2.336 \\
\hline \multicolumn{5}{|c|}{ (ii) Symmetric modes with dominant longitudinal displacements } \\
\hline \multicolumn{2}{|c|}{$S_{1}-S_{2}-S_{3}-\ldots \ldots$} & 4.156 & 5.442 & 5.127 \\
\hline \multicolumn{5}{|c|}{$\begin{array}{l}\text { (iii) Intersections of symmetric and antisymmetric modes } \\
\left(m_{0}, n_{0}\right)\end{array}$} \\
\hline$(1,3)$ & $\begin{array}{l}\mathrm{S}_{2}-\mathrm{A}_{4}-\mathrm{S}_{6}-\ldots \ldots \\
\mathrm{A}_{2}-\mathrm{S}_{4}-\mathrm{A}_{6} \cdots \ldots\end{array}$ & 5.878 & 6.140 & $\begin{array}{l}3.047 \\
3.699\end{array}$ \\
\hline$(1,5)$ & $\begin{array}{l}\mathrm{S}_{3}-\mathrm{A}_{6}-\mathrm{S}_{9}-\ldots \ldots \\
\mathrm{A}_{3}-\mathrm{S}_{6}-\mathrm{A}_{9}-\ldots \ldots\end{array}$ & 10.18 & 5.647 & $\begin{array}{l}3.605 \\
4.478\end{array}$ \\
\hline$(2,4)$ & $\begin{array}{l}\mathrm{A}_{3}-\mathrm{A}_{6}-\mathrm{A}_{9}-\ldots \ldots \\
\mathrm{S}_{3}-\mathrm{S}_{6}-\mathrm{S}_{9}-\ldots \ldots\end{array}$ & 7.199 & 8.314 & $\begin{array}{l}2.583 \\
1.976\end{array}$ \\
\hline$(1,7)$ & $\begin{array}{l}\mathrm{S}_{4}-\mathrm{A}_{8}-\mathrm{S}_{12}-\ldots \ldots \\
\mathrm{A}_{4}-\mathrm{S}_{8}-\mathrm{A}_{12}-\ldots \ldots\end{array}$ & 14.40 & 5.542 & $\begin{array}{l}4.071 \\
4.672\end{array}$ \\
\hline$(3,5)$ & $\begin{array}{l}\mathrm{S}_{4}-\mathrm{A}_{8}-\mathrm{S}_{12}-\ldots \ldots \\
\mathrm{A}_{4}-\mathrm{S}_{8}-\mathrm{A}_{12}-\ldots \ldots\end{array}$ & 8.312 & 28.86 & $\begin{array}{l}0.9934 \\
0.3907\end{array}$ \\
\hline$(1,9)$ & $\begin{array}{l}\mathrm{S}_{5}-\mathrm{A}_{10}-\mathrm{S}_{15^{-}} \ldots \ldots \\
\mathrm{A}_{5}-\mathrm{S}_{10}-\mathrm{A}_{15} \ldots \ldots\end{array}$ & 18.59 & 5.501 & $\begin{array}{l}4.417 \\
4.749\end{array}$ \\
\hline$(2,8)$ & $\begin{array}{l}\mathrm{A}_{5}-\mathrm{A}_{10}-\mathrm{A}_{15^{-}} \ldots \ldots \\
\mathrm{S}_{5}-\mathrm{S}_{10}-\mathrm{S}_{15^{-}} \ldots \ldots\end{array}$ & 16.10 & 5.783 & $\begin{array}{l}3.334 \\
4.243\end{array}$ \\
\hline$(3,7)$ & $\begin{array}{l}\mathrm{S}_{5}-\mathrm{A}_{10}-\mathrm{S}_{15}-\ldots \ldots \\
\mathrm{A}_{5}-\mathrm{S}_{10}-\mathrm{A}_{15}-\ldots \ldots\end{array}$ & 13.14 & 6.944 & $\begin{array}{l}2.815 \\
2.187\end{array}$ \\
\hline$(1,11)$ & $\begin{array}{l}\mathrm{S}_{6}-\mathrm{A}_{12}-\mathrm{S}_{18}-\ldots \ldots \\
\mathrm{A}_{6}-\mathrm{S}_{12}-\mathrm{A}_{18}-\ldots \ldots\end{array}$ & 22.76 & 5.481 & $\begin{array}{l}4.663 \\
4.864\end{array}$ \\
\hline \multicolumn{5}{|c|}{ (iv) Extra Rayleigh modes } \\
\hline$c_{\mathrm{R} 1}$ & $\mathrm{~S}_{1} \mathrm{~A}_{1}-\mathrm{S}_{2} \mathrm{~A}_{2}-\ldots \ldots$ & 2.627 & 6.947 & No matching \\
\hline$c_{\mathrm{R} 2}$ & $\mathrm{~S}_{1} \mathrm{~A}_{1}-\mathrm{S}_{2} \mathrm{~A}_{2}-\ldots \ldots$ & 3.838 & 5.472 & No matching \\
\hline
\end{tabular}

(a)

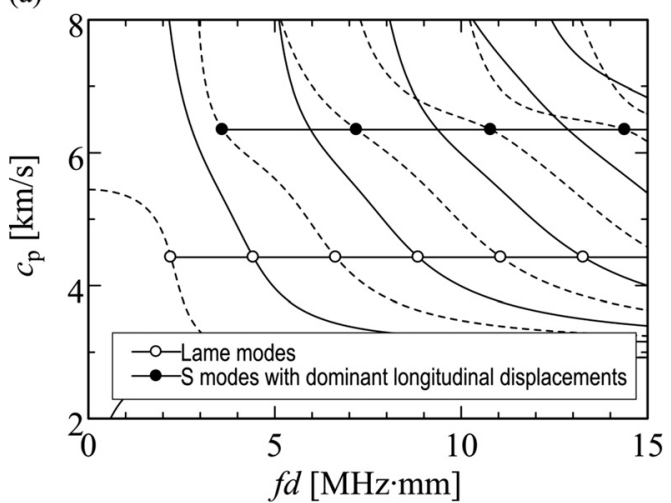

(b)

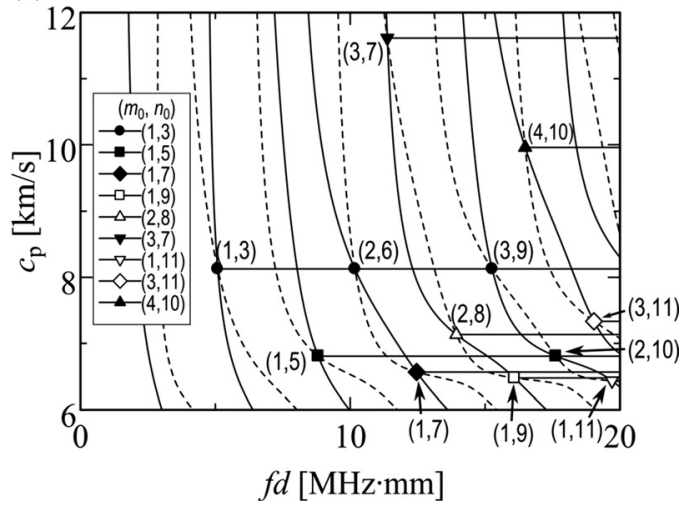

FIG. 2. Lamb modes with phase and group velocity matching on the frequency-phase velocity curves for aluminum (solid lines: A modes, broken lines: S modes). (a) Lamé modes and symmetric modes with dominant longitudinal displacements and (b) intersections of symmetric and antisymmetric modes.

the order not greater than six are shown in Table I. For the mode type (iii), different frequency sets are distinguished by the pair $\left(m_{0}, n_{0}\right)$. For example, when $\left(m_{0}, n_{0}\right)=(1,3)$, the $\mathbf{S}_{2}$, $\mathrm{A}_{4}, \mathrm{~S}_{6}, \ldots$ modes have the phase velocity $8.127 \mathrm{~km} / \mathrm{s}$ and the group velocity $3.766 \mathrm{~km} / \mathrm{s}$ at the $f d$ values of $5.088,10.176$, $15.264, \ldots \mathrm{MHz} \cdot \mathrm{mm}$. At these $f d$ values, the $\mathrm{A}_{2}, \mathrm{~S}_{4}, \mathrm{~A}_{6}, \ldots$ modes, respectively, have the same phase velocity, but their group velocity is $1.952 \mathrm{~km} / \mathrm{s}$.

In Table II, the four mode types (i)-(iv) are shown for an iron plate, with $c_{\mathrm{L}}=5442 \mathrm{~m} / \mathrm{s}$ and $c_{\mathrm{T}}=3303 \mathrm{~m} / \mathrm{s}$, following the example considered by Deng. ${ }^{7}$ This example has $c_{\mathrm{T}} /$ $c_{\mathrm{L}}=0.61 \ldots \ldots>\zeta_{\mathrm{cr}}$, so the extra Rayleigh modes are possible. As described earlier, this type consists of the Lamb modes at intersections of $\mathrm{S}$ and $\mathrm{A}$ modes, such as $\mathrm{S}_{1}-\mathrm{A}_{1}, \mathrm{~S}_{2^{-}}$ $\mathrm{A}_{2}$, and so on.

The modes and frequencies of the Lamb waves which satisfy the phase and group velocity matching are now presented in the $c_{\mathrm{p}}-f d$ diagram. For aluminum, these are shown in Fig. 2(a) for the types (i) and (ii), and in Fig. 2(b) for the type (iii). In Fig. 2(b), different phase velocities are distinguished by the label $\left(m_{0}, n_{0}\right)$ to be easily linked to Table I, and the pair $(m, n)$ for each Lamb mode is attached to the corresponding point of $\left(f d, c_{\mathrm{p}}\right)$. The $c_{\mathrm{g}}-f d$ diagram for aluminum is shown in Fig. 3(a) for the types (i) and (ii), and in Fig. 3(b) and 3(c) for the type (iii). As mentioned in Sec. III, the Lamb modes with the common phase velocity may 
(a)

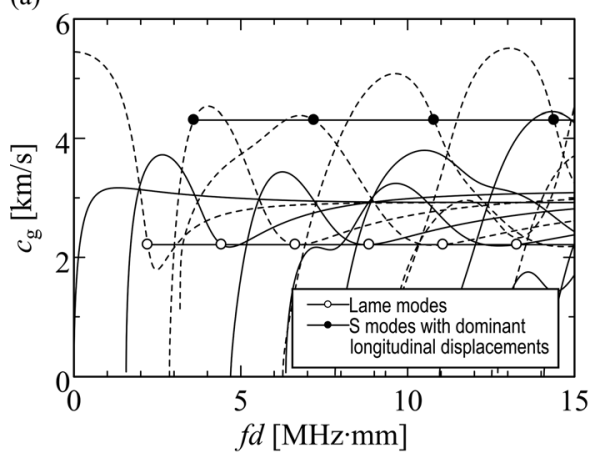

(b)

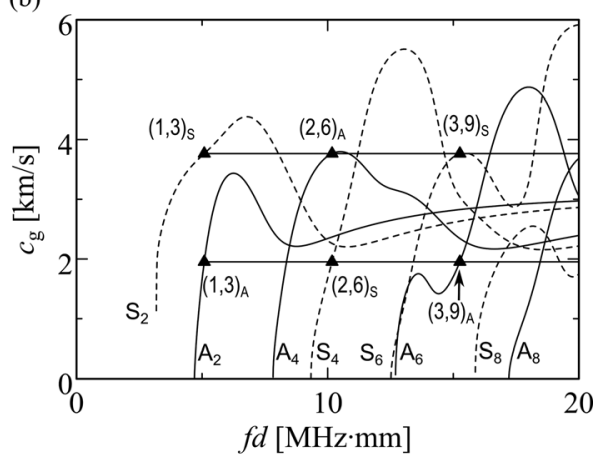

(c)

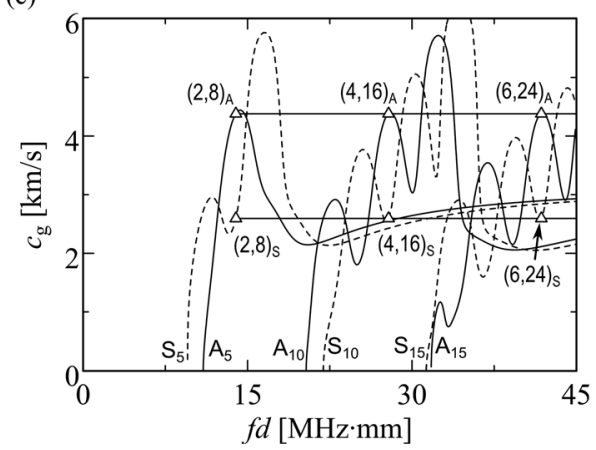

FIG. 3. Lamb modes with phase and group velocity matching on the frequency-group velocity curves for aluminum (solid lines: A modes, broken lines: S modes). (a) Lamé modes and symmetric modes with dominant longitudinal displacements (b) intersections of symmetric and antisymmetric modes when $\left(m_{0}, n_{0}\right)=(1,3)$ and (c) intersections of symmetric and antisymmetric modes when $\left(m_{0}, n_{0}\right)=(2,8)$.

associate two different group velocities in the type (iii). This is illustrated in Fig. 3(b) and 3(c), only for $\left(m_{0}, n_{0}\right)=(1,3)$ and, $(2,8)$ respectively. The subscript $\mathrm{S}$ or $\mathrm{A}$ attached to the label $(m, n)$ denotes the symmetry of the Lamb mode.

For iron, the corresponding $c_{\mathrm{p}}-f d$ and $c_{\mathrm{g}}-f d$ diagrams are shown in Figs. 4 and 5, respectively. In Fig. 4, the four types are separately shown in Fig. 4(a) for the types (i), (ii) and (iv), and in Fig. 4(b) for the type (iii). The three types exhibiting the group velocity matching are shown, in Fig. 5(a) for the types (i) and (ii), and in Figs. 5(b) and 5(c) for the type (iii) with $\left(m_{0}, n_{0}\right)=(1,3)$ and $(2,4)$, respectively. In Fig. 5(d), the group velocities of the Lamb modes in the type (iv) are shown, which take different values for different frequencies. The difference among the group velocities of the $\mathrm{S}$ modes is, however, very small for the case with the (a)

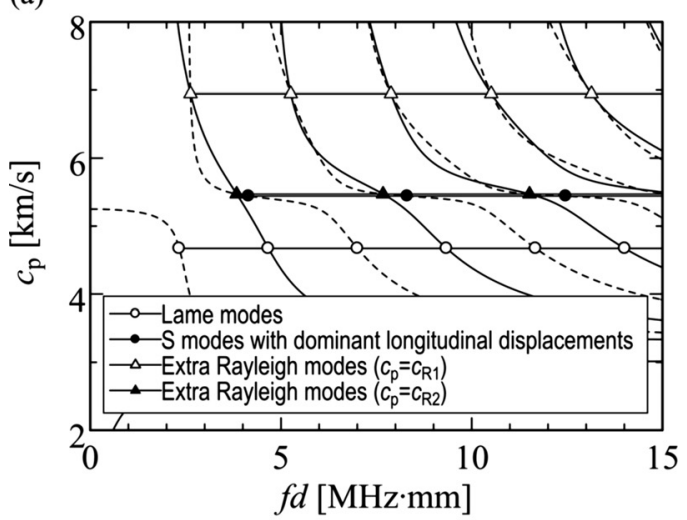

(b)

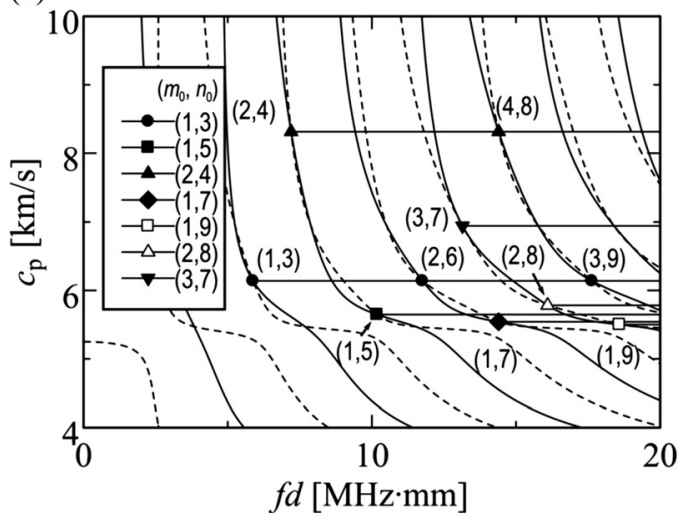

FIG. 4. Lamb modes with phase and group velocity matching on the frequency-phase velocity curves for iron (solid lines: A modes, broken lines: $\mathrm{S}$ modes). (a) Lamé modes, symmetric modes with dominant longitudinal displacements and extra Rayleigh modes, and (b) intersections of symmetric and antisymmetric modes.

phase velocity $c_{\mathrm{R} 2}$. Such near matching of the group velocity can be of equal relevance to the exact matching from a practical point of view.

Figures 2-5 demonstrate the phase and group velocity matching in Lamb waves in a straightforward manner. The frequency and the corresponding velocities for each point in these figures can be readily found in Tables I and II. The finding of the present analysis is thus suitable for the precise determination of the pertinent frequency or the velocity for the cumulative harmonic generation in Lamb waves.

\section{CONCLUDING REMARKS}

In this paper, the phase and group velocity matching between the primary and the harmonic Lamb modes has been analyzed theoretically. By exploiting the RayleighLamb frequency equations in a systematic manner, four mode types have been identified which satisfy matching of the phase velocity, namely, (i) Lamé modes, (ii) symmetric modes with dominant longitudinal displacements, (iii) intersections of symmetric and antisymmetric modes, and (iv) extra Rayleigh modes. The first three types (i)-(iii) have been shown to exhibit the group velocity matching, too. The type (iv) is, however, only possible for a certain range of the transverse to longitudinal wave speeds, and does not exhibit the group velocity matching. It has been also shown 
(a)

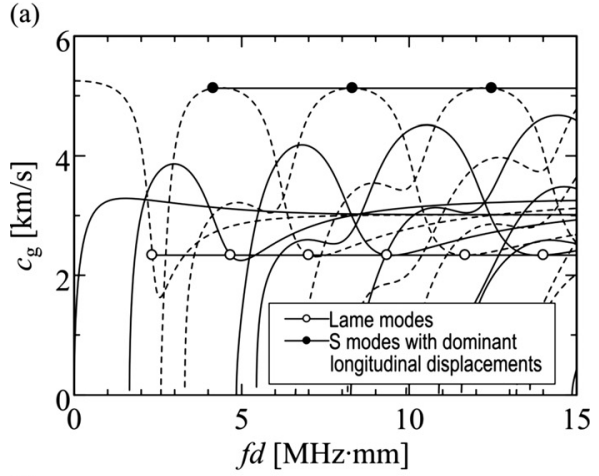

(b)

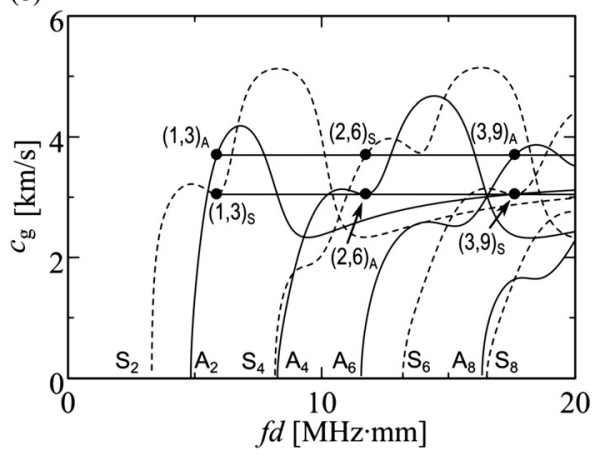

(c)

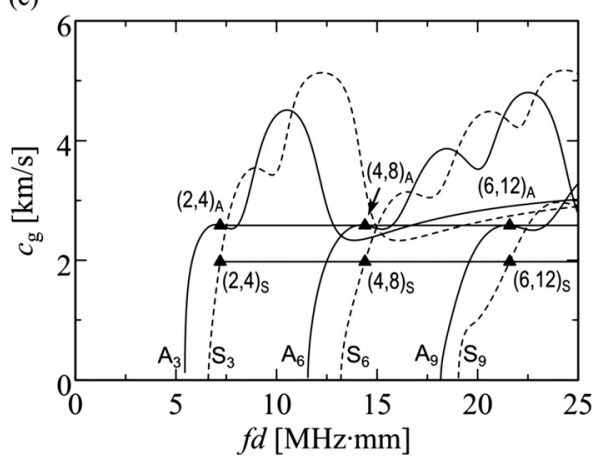

(d)

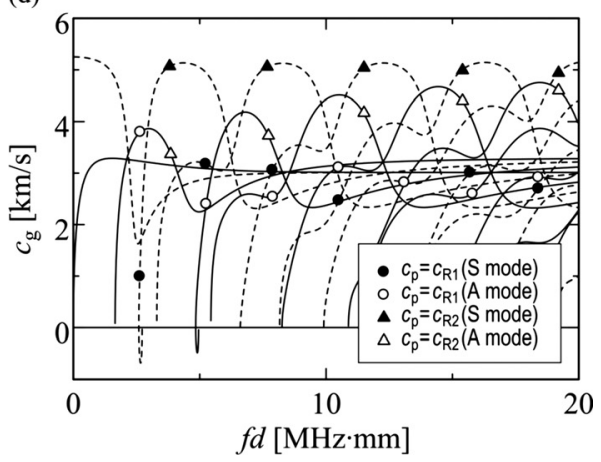

FIG. 5. Lamb modes with phase and group velocity matching on the frequency-group velocity curves for iron (solid lines: A modes, broken lines: S modes). (a) Lamé modes and symmetric modes with dominant longitudinal displacements, (b) intersections of symmetric and antisymmetric modes when $\left(m_{0}, n_{0}\right)=(1,3)$ (c) intersections of symmetric and antisymmetric modes when $\left(m_{0}, n_{0}\right)=(2,4)$ and (d) extra Rayleigh modes.

that the Lamb modes that satisfy the exact phase matching necessarily fall into one of these four types, so the present discussion covers all possibilities of the phase matching in Lamb waves.
The present results have been shown to include those obtained in the previous studies as special examples. From the tabular and graphical demonstrations given in this paper, one can readily find a particular primary Lamb mode and the frequency which exhibit the phase and group velocity matching for cumulative harmonic generation. The surface motions of the relevant Lamb modes have been shown to be purely vertical or horizontal for the types (i)-(iii) and elliptic for the type (iv): such information may be useful in considering experimental arrangements. Among the possible modes and frequencies thus found, however, one needs to select the primary and the harmonic Lamb modes between which nonzero power transfer is possible. The possibility or the efficiency to transfer the energy from the primary to the harmonic modes can be analyzed based on the modal analysis approach established in the foregoing literature. More direct numerical simulations are now in progress by the present authors, which will be presented elsewhere. Supplemented by these approaches, the results in this paper can facilitate practical nonlinear ultrasonic measurements for Lamb waves.

\section{ACKNOWLEDGMENTS}

This investigation has been financially supported by the Japan Society for the Promotion of Science under the Grantin-Aid for Scientific Research (B) (Grant No. 20360053) which is greatly acknowledged.

${ }^{1}$ M. A. Breazeale and D. O. Thompson, Appl. Phys. Lett. 3, 77 (1963).

${ }^{2}$ R. B. Thompson, O. Buck, and D. O. Thompson, J. Acoust. Soc. Am. 59, 1087 (1976).

${ }^{3}$ K.-Y. Jhang and K.-C. Kim, Ultrasonics 37, 39 (1999).

${ }^{4}$ J.-Y. Kim, L. J. Jacobs, J. Qu, and J. W. Littles, J. Acoust. Soc. Am. 120, 1266 (2006).

${ }^{5}$ J. Hermann, J.-Y. Kim, L. J. Jacobs, J. Qu, J. W. Littles, and M. F. Savage, J. Appl. Phys. 99, 124913 (2006).

${ }^{6}$ X. J. Chen, J.-Y. Kim, K. E. Kurtis, J. Qu, C. W. Shen, and L. J. Jacobs, NDT \& E Int. 41, 112 (2008).

${ }^{7}$ M. Deng, J. Appl. Phys. 85, 3051 (1999).

${ }^{8}$ M. Deng, J. Appl. Phys. 94, 4152 (2003).

${ }^{9}$ W. J. N. de Lima and M. F. Hamilton, J. Sound Vib. 265, 819 (2003).

${ }^{10}$ M. Deng, P. Wang, and X. Lv, Appl. Phys. Lett. 86, 124104 (2005).

${ }^{11}$ C. Bermes, J.-Y. Kim, J. Qu, and L. J. Jacobs, Appl. Phys. Lett. 90, 021901 (2007).

${ }^{12}$ C. Pruell, J.-Y. Kim, J. Qu, and L. J. Jacobs, Appl. Phys. Lett. 91, 231911 (2007).

${ }^{13}$ A. Srivastava and F. Lanza di Scalia, J. Sound Vib. 323, 932 (2009).

${ }^{14}$ T.-H. Lee, I.-H. Choi, and K.-Y. Jhang, Mod. Phys. Lett. B 22, 1135 (2008).

${ }^{15}$ C. Pruell, J.-Y Kim, J. Qu, and L. J. Jacobs, Smart Mater. Struct. 18, 035003 (2009).

${ }^{16}$ M. F. Müller, J.-Y. Kim, J. Qu, and L. J. Jacobs, J. Acoust. Soc. Am. 127, 2141 (2010).

${ }^{17}$ K. F. Graff, Wave Motion in Elastic Solids (Oxford University Press, London, 1975).

${ }^{18}$ A. Pilarski, J. J. Ditri, and J. L. Rose, J. Acoust. Soc. Am. 93, 2228 (1993).

${ }^{19}$ J. L. Rose, Ultrasonic Waves in Solid Media (Cambridge University Press, Cambridge, 1999).

${ }^{20}$ K. H. Matlack, J.-Y. Kim, L. J. Jacobs, and J. Qu, J. Appl. Phys. 109, 014905 (2011). 